\title{
THE LOGARITHMIC SKEW-NORMAL DISTRIBUTIONS ARE MOMENT-INDETERMINATE
}

\author{
GWO DONG LIN, ${ }^{*}$ Academia Sinica, Taipei \\ JORDAN STOYANOV, ${ }^{* *}$ Newcastle University
}

\begin{abstract}
We study the class of logarithmic skew-normal (LSN) distributions. They have heavy tails; however, all their moments of positive integer orders are finite. We are interested in the problem of moments for such distributions. We show that the LSN distributions are all nonunique (moment-indeterminate). Moreover, we explicitly describe Stieltjes classes for some LSN distributions; they are families of infinitely many distributions, which are different but have the same moment sequence as a fixed LSN distribution.
\end{abstract}

Keywords: Logarithmic skew-normal distribution; heavy tail; finite moments; problem of moments; nonuniqueness; Stieltjes class

2000 Mathematics Subject Classification: Primary 60E05

Secondary 44A60

\section{Introduction}

The normal and lognormal distributions occupy a central position in both the theory and applications of probability and statistics. However, when modelling complex random phenomena in applied areas, there was a real need of more flexible distributions which were beyond the normal; see, e.g. O'Hagan and Leonard (1976), Azzalini (1985), or Schmoyeri et al. (1996). This is why the class of skew-normal distributions was introduced in Azzalini (1985) and intensively studied by many authors.

In this paper we focus on the class of logarithmic skew-normal distributions and investigate their moment determinacy. It is worth mentioning that these distributions have been used recently in Chai and Bailey (2008) to analyze the continuous (coronary artery calcification) data in a two-part stochastic model.

In Section 2 we present basic properties of logarithmic skew-normal distributions, including a probabilistic representation. In Section 3 we study the problem of moments for this class of distributions, showing that they are all moment-indeterminate. This extends the classical result for moment-indeterminacy of the lognormal distribution given in Stieltjes (1894) and developed in Heyde (1963). In Section 4 we present explicit Stieltjes classes for some logarithmic skewnormal distributions. Finally, some possible extensions of the main results are briefly discussed in Section 5.

Received 15 October 2008; revision received 16 June 2009.

* Postal address: Institute of Statistical Science, Academia Sinica, Taipei 11529, Taiwan, Republic of China.

** Postal address: School of Mathematics and Statistics, Newcastle University, Newcastle upon Tyne, NE1 7RU, UK.

Email address: jordan.stoyanov@ncl.ac.uk

Dedicated to the memory of Professor Chris Heyde (1939-2008). 


\section{Logarithmic skew-normal distributions: basic properties}

We use $\phi$ and $\Phi$ to denote the standard normal density function and the standard normal distribution function, respectively. For any constant $\lambda \in \mathbb{R}=(-\infty, \infty)$, consider the random variable $X_{\lambda}$ obeying a skew-normal distribution (denoted by $\mathrm{SN}(\lambda)$ ), i.e. $X_{\lambda} \sim \mathrm{SN}(\lambda)$. The density function $f_{\lambda}$ of $X_{\lambda}$ is $f_{\lambda}(x)=2 \phi(x) \Phi(\lambda x), x \in \mathbb{R}$. To see that $f_{\lambda}$ is a bona fide density function, we have, by the symmetry property of $\Phi, \int_{-\infty}^{\infty} f_{\lambda}(x) \mathrm{d} x=2 \int_{0}^{\infty} \phi(x) \mathrm{d} x=1$.

Definition. We say that the positive random variable $Y_{\lambda}$ has a logarithmic skew-normal distribution, and write $Y_{\lambda} \sim \operatorname{LSN}(\lambda)$, if its logarithmic transform $\ln Y_{\lambda} \sim \operatorname{SN}(\lambda)$. The density function of $Y_{\lambda}$, denoted by $g_{\lambda}$, is given by:

$$
g_{\lambda}(y)=\frac{2}{y} \phi(\ln y) \Phi(\lambda \ln y), \quad y>0 .
$$

The skew parameter $\lambda$ regulates the shape of the distribution. If $\lambda=0, \operatorname{LSN}(\lambda)$ reduces to the standard lognormal distribution $\operatorname{LN}(0,1)$.

To derive some basic properties of LSN distributions, we need the following result from Arnold and Lin (2004).

Lemma 1. (Arnold and Lin (2004, Lemma 2, p. 598).) The standard normal distribution function $\Phi$ obeys the property $-\ln \Phi(-x)=O\left(x^{2}\right)$ as $x \rightarrow \infty$. More precisely,

$$
\lim _{x \rightarrow \infty} \frac{-\ln \Phi(-x)}{x^{2}}=\frac{1}{2} \text {. }
$$

The next statement shows that the LSN distributions have heavy tails.

Proposition 1. For any $\lambda \in \mathbb{R}$, the random variable $Y_{\lambda} \sim \operatorname{LSN}(\lambda)$ does not have a moment generating function. Equivalently, $\mathrm{E}\left[\mathrm{e}^{t Y_{\lambda}}\right]=\infty$ for each $t>0$.

Proof. Using the expression for the density $g_{\lambda}$, (1), we obtain

$$
\mathrm{E}\left[\mathrm{e}^{t Y_{\lambda}}\right]=\int_{0}^{\infty} \mathrm{e}^{t y} g_{\lambda}(y) \mathrm{d} y=\int_{0}^{\infty} \frac{2}{y} \mathrm{e}^{t y} \phi(\ln y) \Phi(\lambda \ln y) \mathrm{d} y=\int_{0}^{\infty} h_{\lambda}(y) \mathrm{d} y .
$$

Here $t>0$ is fixed and $h_{\lambda}(y):=(2 / y) \mathrm{e}^{t y} \phi(\ln y) \Phi(\lambda \ln y)>0$ for all $y>0$. We want to show that $J_{\lambda}:=\int_{0}^{\infty} h_{\lambda}(y) \mathrm{d} y=\infty$ for any $\lambda \in \mathbb{R}$.

Indeed, for $\lambda \geq 0$, we have $\liminf _{y \rightarrow \infty} \Phi(\lambda \ln y) \geq \frac{1}{2}$. This implies that $h_{\lambda}(y) \rightarrow \infty$ as $y \rightarrow \infty$; hence, in this case $J_{\lambda}=\infty$.

Then, for $\lambda<0$, we have, by Lemma 1 , the asymptotic equivalence

$$
\ln \Phi(\lambda \ln y) \simeq-\frac{1}{2}(\lambda \ln y)^{2} \quad \text { as } y \rightarrow \infty,
$$

which implies that

$$
\ln h_{\lambda}(y) \simeq \frac{1}{2} \ln \left(\frac{2}{\pi}\right)-\ln y+t y-\frac{1}{2}\left(\lambda^{2}+1\right)(\ln y)^{2} \rightarrow \infty \quad \text { as } y \rightarrow \infty .
$$

Hence, also in this case, $J_{\lambda}=\infty$. This completes the proof.

In general, the existence of the moment generating function implies that all moments of positive integer orders are finite. However, there are distributions that do not have moment generating functions, but still have finite moments of any positive order; see, e.g. Stoyanov (1997, Sections 8 and 11). This is exactly the case for LSN distributions. 
Proposition 2. For any $\lambda \in \mathbb{R}$, the random variable $Y_{\lambda} \sim \operatorname{LSN}(\lambda)$ has finite moments of all positive integer orders. Equivalently, $\mathrm{E}\left[Y_{\lambda}^{k}\right]<\infty$ for any positive integer $k$.

In the next proposition we give an explicit formula for the moment $\mathrm{E}\left[Y_{\lambda}^{t}\right]=\mathrm{E}\left[\mathrm{e}^{t X_{\lambda}}\right]$ for any $t \in \mathbb{R}$, not just for positive integers $k$; see, e.g. Azzalini (1985, p. 174).

Proposition 3. Let $Y_{\lambda} \sim \operatorname{LSN}(\lambda)$ for some $\lambda \in \mathbb{R}$. Then, for any real $t$, the $t$-order moment $\mathrm{E}\left[Y_{\lambda}^{t}\right]$ is given by

$$
m_{t}(\lambda):=\mathrm{E}\left[Y_{\lambda}^{t}\right]=\mathrm{E}\left[\mathrm{e}^{t X_{\lambda}}\right]=2 \mathrm{e}^{t^{2} / 2} \Phi(\delta t), \text { where } \delta=\frac{\lambda}{\sqrt{1+\lambda^{2}}} .
$$

Proof. The proof follows directly by checking that

$$
\frac{\mathrm{d}}{\mathrm{d} \lambda} m_{t}(\lambda)=2 \mathrm{e}^{t^{2} / 2} \phi(\delta t) \frac{\mathrm{d}}{\mathrm{d} \lambda}(\delta t) .
$$

We next give a probabilistic representation for an LSN random variable.

Proposition 4. Any LSN random variable is distributionally equivalent to a product of two independent random variables, one which is lognormal and one which is log-half-normal. Specifically, for any $\lambda \in \mathbb{R}$, the random variable $Y_{\lambda} \sim \operatorname{LSN}(\lambda)$ has the following representation:

$$
Y_{\lambda} \stackrel{\mathrm{D}}{=} \mathrm{e}^{\delta\left|Z_{1}\right|} \exp \left[\sqrt{1-\delta^{2}} Z_{2}\right], \quad \text { where } \delta=\frac{\lambda}{\sqrt{1+\lambda^{2}}},
$$

$Z_{1}$ and $Z_{2}$ are two independent standard normal random variables, and 'D ' denotes equality in distribution.

The proof of Proposition 4 is based on the probabilistic representation of any skew-normal random variable as a linear combination of two independent random variables: one standard normal and one standard half-normal (see Henze (1986)).

From the above we know that $Y_{\lambda} \sim \operatorname{LSN}(\lambda)$ has all finite moments; hence, we can turn to one of the main questions discussed in this work: is the distribution of $Y_{\lambda}$ uniquely determined by its moments? The answer is presented in the next section.

\section{The problem of moments for LSN distributions}

Suppose that a random variable $\xi$ with distribution function $F$ on the real line has finite moments, $\left\{\mathrm{E}\left[\xi^{k}\right]\right\}$, for all positive integer orders $k$. If $F$ is uniquely determined by the moment sequence $\left\{\mathrm{E}\left[\xi^{k}\right]\right\}_{k=1}^{\infty}$, we say that $\xi$, and also $F$, is unique, or moment-determinate (M-determinate). Otherwise, $\xi$, and also $F$, is M-indeterminate.

In general, if $\xi$ has a moment generating function, not only are all moments finite, but $F$ is also unique, or M-determinate. Note, however, that there are heavy-tailed distributions, i.e. without moment generating functions, such that all their moments are finite and the distributions are M-determinate; see, e.g. Stoyanov (1997, Section 11).

Since, by Proposition 3, we explicitly know the moments of $Y_{\lambda} \sim \operatorname{LSN}(\lambda)$, an obvious idea is to try to use the well-known Carleman criterion. Recall that if $F$ is a distribution on the interval $(0, \infty)$ with finite moments $m_{k}=\int_{0}^{\infty} x^{k} \mathrm{~d} F(x), k=1,2, \ldots$, and we calculate the Carleman quantity

$$
\mathrm{C}\left[\left\{m_{k}\right\}\right]:=\sum_{k=1}^{\infty}\left(m_{k}\right)^{-1 /(2 k)},
$$


then the condition $\mathrm{C}=\infty$ is sufficient for $F$ to be M-determinate. In other words, $F$ is the only distribution with the moment sequence $\left\{m_{k}\right\}$. There are, however, M-determinate distributions for which the Carleman quantity C is finite; see, e.g. Stoyanov (1997, Section 11). In all cases, if $F$ is $\mathrm{M}$-indeterminate then $\mathrm{C}<\infty$ necessarily.

In our case $m_{k}(\lambda)=\mathrm{E}\left[Y_{\lambda}^{k}\right]=2 \mathrm{e}^{k^{2} / 2} \Phi(k \delta), k=1,2, \ldots$, so we need to analyze the Carleman quantity $\mathrm{C}=\mathrm{C}(\lambda)$, which depends on $\lambda$ through $\delta=\lambda / \sqrt{1+\lambda^{2}}$. We have

$$
\mathrm{C}(\lambda):=\mathrm{C}\left[\left\{m_{k}(\lambda)\right\}\right]=\sum_{k=1}^{\infty}\left(m_{k}(\lambda)\right)^{-1 /(2 k)}=\sum_{k=1}^{\infty}\left(2 \mathrm{e}^{k^{2} / 2} \Phi(k \delta)\right)^{-1 /(2 k)}=\sum_{k=1}^{\infty} c_{k} \mathrm{e}^{-k / 4},
$$

where $c_{k}=(2 \Phi(k \delta))^{-1 /(2 k)}$. Since

$$
\lim _{k \rightarrow \infty} c_{k}=1 \quad \text { for } \lambda \geq 0 \text { and } \quad \lim _{k \rightarrow \infty} \frac{\ln c_{k}}{k \delta^{2} / 4}=1 \text { for } \lambda<0,
$$

it immediately follows that $\mathrm{C}(\lambda)<\infty$ for any $\lambda \in \mathbb{R}$. Hence, the Carleman criterion does not hold, although it perhaps suggests that $Y_{\lambda}$ is M-indeterminate. This is the statement of Theorem 1, below, and its proof is based on a different idea.

It is well known that the standard lognormal distribution is M-indeterminate. This result, in a slightly different form, is due to Stieltjes (1894). Heyde (1963) extended the result to general lognormal distributions and his analysis was given in modern probabilistic/statistical terms. See also Stoyanov (1997, Section 11) and Stoyanov (2000). As mentioned before, the random variable $Y_{\lambda} \sim \operatorname{LSN}(\lambda)$ with $\lambda=0$ has a standard lognormal distribution, $\operatorname{LN}(0,1)$. It is one of our aims in this paper to show that, for any $\lambda \in \mathbb{R}$, the $\operatorname{LSN}$ distributions, $\operatorname{LSN}(\lambda)$, share the same moment indeterminacy property as that of the standard lognormal distribution.

Theorem 1. For any $\lambda \in \mathbb{R}$, the random variable $Y_{\lambda}$ is $M$-indeterminate.

Proof. We use the Krein criterion (see, e.g. Akhiezer (1965, p. 87), Slud (1993), Lin (1997), Stoyanov (2000), or Pakes et al. (2001)). So we need to calculate the Krein quantity, $\mathrm{K}\left[g_{\lambda}\right]$, where

$$
\mathrm{K}\left[g_{\lambda}\right]:=\int_{0}^{\infty} \frac{-\ln g_{\lambda}\left(y^{2}\right)}{1+y^{2}} \mathrm{~d} y .
$$

For the M-indeterminacy of $Y_{\lambda}$, it suffices to show that $\mathrm{K}\left[g_{\lambda}\right]<\infty$ for $\lambda \in \mathbb{R}$. By (1),

$$
\mathrm{K}\left[g_{\lambda}\right]=I_{1}+I_{2}+I_{3}+I_{4},
$$

where

$$
\begin{aligned}
I_{1} & =\int_{0}^{\infty} \frac{-\ln 2}{1+y^{2}} \mathrm{~d} y, & I_{2} & =\int_{0}^{\infty} \frac{2 \ln y}{1+y^{2}} \mathrm{~d} y, \\
I_{3} & =\int_{0}^{\infty} \frac{-\ln \phi(2 \ln y)}{1+y^{2}} \mathrm{~d} y, & I_{4} & =\int_{0}^{\infty} \frac{-\ln \Phi(2 \lambda \ln y)}{1+y^{2}} \mathrm{~d} y .
\end{aligned}
$$

Integrals $I_{1}-I_{4}$ can easily be seen to be finite by checking, by means of Lemma 1 , that, for a finite positive constant $c$,

$$
|\ln \phi(2 \ln y)|+|\ln \Phi(2 \lambda \ln y)| \leq c\left(1+(\ln y)^{2}\right), \quad y>0,
$$

and that, for $r=0,1,2$, we have

$$
\int_{0}^{\infty} \frac{|\ln y|^{r}}{1+y^{2}} \mathrm{~d} y<\infty
$$


Theorem 1 exhibits the moment indeterminacy of $Y_{\lambda} \sim \operatorname{LSN}(\lambda)$ for any fixed $\lambda$. It is, therefore, interesting to clarify what happens if $\lambda \rightarrow+\infty$ or $\lambda \rightarrow-\infty$.

Theorem 2. Let $Z$ be a standard normal random variable, $Z \sim N(0,1)$, and define $\eta=\mathrm{e}^{|Z|}$, usually called the log-half-normal random variable. Then, denoting by $\stackrel{\mathrm{D}}{\rightarrow}$ convergence in distribution, the following properties hold.

(a) $Y_{\lambda} \stackrel{\mathrm{D}}{\rightarrow} \eta$ as $\lambda \rightarrow+\infty$ and $Y_{\lambda} \stackrel{\mathrm{D}}{\rightarrow} 1 / \eta$ as $\lambda \rightarrow-\infty$.

(b) The left limit $1 / \eta$ is M-determinate, while the right limit $\eta$ is M-indeterminate.

(c) For $t>0, m_{t}(\lambda)=\mathrm{E}\left[Y_{\lambda}^{t}\right]$ is an increasing function of $\lambda \in \mathbb{R}$, while $m_{t}(\lambda)$ is decreasing in $\lambda$ for $t<0$.

(d) For any $k=1,2, \ldots$, we have

$$
\begin{aligned}
& \lim _{\lambda \rightarrow+\infty} m_{k}(\lambda)=2 \mathrm{e}^{k^{2} / 2} \Phi(k)=\mathrm{E}\left[\mathrm{e}^{k|Z|}\right]=\mathrm{E}\left[\eta^{k}\right], \\
& \lim _{\lambda \rightarrow-\infty} m_{k}(\lambda)=2 \mathrm{e}^{k^{2} / 2} \Phi(-k)=\mathrm{E}\left[\mathrm{e}^{-k|Z|}\right]=\mathrm{E}\left[\left(\frac{1}{\eta}\right)^{k}\right] .
\end{aligned}
$$

Proof. Claim (a) follows immediately from the probabilistic representation of $Y_{\lambda}$ given in Proposition 4. We can also use the definitions of $Z, X_{\lambda}$, and $Y_{\lambda}$, and apply Slutsky's theorem to conclude first that $X_{\lambda} \stackrel{\mathrm{D}}{\rightarrow}|Z|$ as $\lambda \rightarrow+\infty$ and $X_{\lambda} \stackrel{\mathrm{D}}{\rightarrow}-|Z|$ as $\lambda \rightarrow-\infty$, and then that $Y_{\lambda} \stackrel{\mathrm{D}}{\rightarrow} \eta$ as $\lambda \rightarrow+\infty$ and $Y_{\lambda} \stackrel{\mathrm{D}}{\rightarrow} 1 / \eta$ as $\lambda \rightarrow-\infty$. For claim (b), we first note that the random variable $1 / \eta=\mathrm{e}^{-|Z|}$ is bounded; hence, it is M-determinate. On the other hand, the Krein quantity for $\eta$ is finite, which also tells us that $\eta$ is M-indeterminate. Finally, the proofs of claims (c) and (d) are based on elementary properties of the normal distribution function and are thus omitted.

\section{Stieltjes classes for LSN distributions}

We start with a distribution function $F=\operatorname{LSN}(\lambda), \lambda \geq 0$, and we let $f$ denote the density function, instead of $g_{\lambda}$ as in (1).

In general, a Stieltjes class for an M-indeterminate distribution, e.g. $F$, is a parameterized family of different distributions all having the same moments as $F$; see Stoyanov (2004). In the absolutely continuous case, the Stieltjes class, denoted by $S$, is defined in terms of $f$ and another function $p$, which is called a perturbation function:

$$
S=S(f, p)=\left\{f_{\varepsilon}: f_{\varepsilon}(x)=f(x)[1+\varepsilon p(x)], x \in \mathbb{R}, \varepsilon \in[-1,1]\right\} .
$$

Here $p$ is a measurable function with $|p(x)| \leq 1$ for all $x \in \mathbb{R}$. The density $f$ and the perturbation $p$ are such that the product function $v(x):=f(x) p(x), x \in \mathbb{R}$, has vanishing 'moments' in the sense that $\int_{-\infty}^{\infty} x^{k} v(x) \mathrm{d} x=0$ for any $k=0,1,2, \ldots$

To construct a Stieltjes class for the $\operatorname{LSN}(\lambda)$ distribution with $\lambda \geq 0$, we need some preparation. First, consider the function $\tilde{p}=(\tilde{p}(x), x \in \mathbb{R})$, defined as follows:

$$
\tilde{p}(x)= \begin{cases}\left(\frac{x}{x-1}\right)^{1+\ln x(x-1) / 2} \frac{\sin [\pi \ln (x-1)]}{\Phi(\lambda \ln x)}=\frac{\ell(x-1) \frac{\sin [\pi \ln (x-1)]}{\Phi(\lambda \ln x)}}{\ell(x)} & \text { if } x>1, \\ 0 & \text { if } x \leq 1 .\end{cases}
$$


Here $\ell$ is the lognormal $\operatorname{LN}(0,1)$ density:

$$
\ell(x)=\frac{1}{\sqrt{2 \pi}} \frac{1}{x} \exp \left[-\frac{1}{2}(\ln x)^{2}\right], \quad x>0 ; \quad \ell(x)=0, \quad x \leq 0 .
$$

This kind of function with $\lambda=0$ has been considered in Stoyanov and Tolmatz (2005). Here we need a property of $\tilde{p}$.

Lemma 2. For any $\lambda \geq 0$, the function $\tilde{p}$ is bounded, i.e. there is a positive constant $\tilde{c}$ such that $|\tilde{p}(x)| \leq \tilde{c}<\infty$ for all $x \in \mathbb{R}$.

Proof. To show the validity of the statement, we need the following three facts.

Fact 1. The function $\tilde{p}$ is continuous on the interval $(1, \infty)$.

Fact 2. At the boundary points 1 and $\infty$, we have

$$
\lim _{x \rightarrow 1^{+}} \frac{\ell(x-1)}{\ell(x)}=0, \quad \lim _{x \rightarrow \infty} \frac{\ell(x-1)}{\ell(x)}=1 .
$$

Fact 3. For any $\lambda \geq 0$ and $x>1$, we have $\frac{1}{2} \leq \Phi(\lambda \ln x) \leq 1$.

The statement of the lemma now easily follows from facts 1-3.

Theorem 3. Suppose that $X$ is a random variable and that $X \sim \operatorname{LSN}(\lambda)$ for some $\lambda \geq 0$. Denote by $F$ the distribution function of $X$, and let $f$ be its density function (see (1)):

$$
f(x)=f(x \mid \lambda)=\frac{2}{x} \phi(\ln x) \Phi(\lambda \ln x), \quad x>0 .
$$

Furthermore, define the function $p=(p(x), x \in \mathbb{R})$ as follows:

$$
p(x)=p(x \mid \lambda)=\frac{1}{\tilde{c}} \tilde{p}(x), \quad x \in \mathbb{R},
$$

where $\tilde{p}$ is given by (2) and $\tilde{c}$ is the constant in Lemma 2. Then the family of functions

$$
S=S(f, p)=\left\{f_{\varepsilon}: f_{\varepsilon}(x)=f(x)[1+\varepsilon p(x)], x \in \mathbb{R}, \varepsilon \in[-1,1]\right\}
$$

is a Stieltjes class for the M-indeterminate distribution $F$.

Proof. The crucial point is to show that the product function $v:=f p$ has vanishing moments, i.e. that $\int_{0}^{\infty} x^{k} f(x) p(x) \mathrm{d} x=0$ for any $k=0,1,2, \ldots$ We use the function

$$
v_{0}(x)=\frac{1}{x} \exp \left[-\frac{1}{2}(\ln x)^{2}\right] \sin (\pi \ln x), \quad x>0,
$$

and the property that $\int_{0}^{\infty} x^{k} v_{0}(x) \mathrm{d} x=0$ for any $k=0,1,2, \ldots$ Details can be found in Heyde (1963) or Gradshteyn and Ryzhik (2000, Formula 4.133); see also Stoyanov (1997, Section 11). In other words, $v_{0}(x), x>0$, is a function with vanishing moments. Then the shifted function $\tilde{v}(x):=v_{0}(x-1), x>1$, also has vanishing moments. Finally, we conclude that the product function $v(x)=f(x) p(x)=c \tilde{v}(x), x>1$, has vanishing moments. This completes the proof. 
Remark. We can use the same M-indeterminate distribution $F$ and its density $f$ in combination with other perturbations $p$ to construct new Stieltjes classes, which are different from those mentioned above. Results from Stoyanov (2004) and Stoyanov and Tolmatz (2005) can easily be adapted to LSN distributions. Moreover, for any Stieltjes class, we can introduce the socalled index of dissimilarity, which is a measure of how much the distribution functions in this class differ. Details can be found in Stoyanov (2004).

\section{Possible extensions}

1. Knowing that any random variable $Y_{\lambda} \sim \operatorname{LSN}(\lambda)$ is M-indeterminate, we can easily show that some nonlinear transformations of $Y_{\lambda}$, called Box-Cox transformations, are also Mindeterminate. In particular, for any nonzero real $r$, the power $\left(Y_{\lambda}\right)^{r}$ is M-indeterminate. Also, the Stieltjes class in Theorem 3 can be adapted to this case with $r \lambda>0$ and $r^{2}$ integer.

2. The skew-normal distribution, $\mathrm{SN}(\lambda)$, is in fact a conditional distribution in the hidden truncation model described below.

Let $\left(Z_{1}, Z_{2}\right)$ be a bivariate normally distributed random vector with mean $(0,0)$, variances $\operatorname{var}\left(Z_{1}\right)=\operatorname{var}\left(Z_{2}\right)=1$, and correlation coefficient $\rho \in(-1,1)$. Then the conditional distribution of $Z_{1}$, given $Z_{2}>0$ (usually ignored and, thus, called a hidden truncation at the mean), is $\operatorname{SN}(\lambda(\rho))$, where $\lambda(\rho)=\rho / \sqrt{1-\rho^{2}}$ (see, e.g. Azzalini and Dalla Valle (1996)). In general, for any $a \in \mathbb{R}$, the conditional distribution of $Z_{1}$, given $Z_{2}>a$ (a hidden truncation at $a$ ), has density function

$$
f_{a, \lambda}(x)=[\Phi(-a)]^{-1} \phi(x) \Phi\left(\frac{-a}{\sqrt{1-\rho^{2}}}+\lambda(\rho) x\right), \quad x \in \mathbb{R}
$$

(see, e.g. Arnold and Beaver (2000)). Let $Y_{a, \lambda}$ be a positive random variable such that $\ln Y_{a, \lambda}$ has the density function $f_{a, \lambda}$ in (3). Then $Y_{a, \lambda}$ is M-indeterminate. This will be an extension of our Theorem 1. The proof follows the same steps and the details are thus omitted. The Stieltjes class in Theorem 3 can be adapted to this case with $\rho>0$.

3. Suppose that in the original definition of the skew-normal density $2 \phi(x) \Phi(\lambda x), x \in \mathbb{R}$, we replace the normal density $\phi$ by an arbitrary symmetric density $g(x), x \in \mathbb{R}$, and the standard normal distribution function $\Phi$ by an arbitrary symmetric distribution function $H(x), x \in \mathbb{R}$. Both symmetries are with respect to 0 . Then, for $\lambda \in \mathbb{R}$, we define the function $f(x \mid \lambda)=2 g(x) H(\lambda x), x \in \mathbb{R}$, which is a density. The distribution $F$ with density $f$ is said to be a skew-symmetric distribution. A positive random variable $Y$ is said to obey a logarithmic skew-symmetric distribution if its logarithmic transform $\ln Y$ has a skew-symmetric distribution. It is possible to find conditions on the density $g$ and the distribution $H$ such that $Y$ is M-indeterminate. This also extends our Theorem 1. Details will be reported elsewhere.

4. We suggest that the class of LSN distributions is linked to stochastic models involving distributions which are more flexible than the normal and lognormal distributions. Besides Chai and Bailey's (2008) paper cited in the introduction, other relevant references include Azzalini et al. (2003), Williamson and Gaston (2005), and Yamazaki and Lueck (1990). 


\section{Acknowledgements}

This work commenced during the visit of the second author to the Institute of Statistical Science, Academia Sinica, Taipei. He thanks his Taiwanese colleagues for their hospitality and the Institute for their support. The work of the first author was partly supported by the National Science Council of Taiwan, Republic of China, under grant NSC 96-2628-M-001-022.

The authors are grateful to the anonymous referee and the Editor for their constructive suggestions and comments. In particular, the final part of the elegant proof of Theorem 1 was suggested by the referee.

\section{References}

Akhiezer, N. I. (1965). The Classical Moment Problem and Some Related Questions in Analysis. Hafner Publishing, New York.

Arnold, B. C. And Beaver, R. J. (2000). Hidden truncation models. Sankhyā A 62, 23-35.

ARnOLD, B. C. AND Lin, G. D. (2004). Characterizations of the skew-normal and generalized chi distributions. Sankhya 66, 593-606.

Azzalini, A. (1985). A class of distributions which includes the normal ones. Scand. J. Statist. 12, 171-178.

Azzalini, A., Dal Cappello, T. And Kotz, S. (2003). Log-skew-normal and log-skew- $t$ distributions as models for family income data. J. Income Distribution 11, 12-20.

Azzalini, A. and Dalla Valle, A. (1996). The multivariate skew-normal distribution. Biometrika 83, 715-726.

Chai, H. S. AND BAILEy, K. R. (2008). Use of log-skew-normal distribution in analysis of continuous data with a discrete component at zero. Statist. Med. 27, 3643-3655.

Gradshteyn, I. S. And RyzhiK, I. M. (2000). Tables of Integrals, Series, and Products, 6th edn. Academic Press, San Diego, CA.

HenZe, N. (1986). A probabilistic representation of the 'skew-normal' distribution. Scand. J. Statist. 13, $271-275$.

Heyde, C. C. (1963). On a property of the lognormal distribution. J. R. Statist. Soc. B 25, 392-393.

LIN, G. D. (1997). On the moment problems. Statist. Prob. Lett. 35, 85-90. (Correction: 50 (2000), 205.)

O'HaGAn, A. AND LeONARD, T. (1976). Bayes estimation subject to uncertainty about parameter constraints. Biometrika 63, 201-203.

PAKes, A., Hung, W.-L. AND Wu, J.-W. (2001). Criteria for the unique determination of probability distributions by moments. Austral. N. Z. J. Statist. 43, 101-111.

Schmoyeri, R. L., Beauchamp, J. J., Brandt, C. C. and Hoffman, F. O. JR. (1996). Difficulties with the lognormal model in mean estimation and testing. Environm. Ecol. Statist. 3, 81-97.

SLud, E. V. (1993). The moment problem for polynomial forms in normal random variables. Ann. Prob. 21, $2200-2214$.

Stieltjes, T. J. (1894). Recherches sur les fractions continues. Ann. Fac. Sci. Univ. Toulouse 8, J1-J122 and 9 (1895), A5-A47. Reprinted in: Ann. Fac. Sci. Univ. Toulouse (6) 4 (1995), A5-A47.

Stoyanov, J. (1997). Counterexamples in Probability, 2nd edn. John Wiley, Chichester.

Stoyanov, J. (2000). Krein condition in probabilistic moment problems. Bernoulli 6, 939-949.

Stoyanov, J. (2004). Stieltjes classes for moment-indeterminate probability distributions. In Stochastic Methods and Their Applications (J. Appl. Prob. Spec. Vol. 41A), eds J. Gani and E. Seneta, Applied Probability Trust, Sheffield, pp. 281-294.

Stoyanov, J. And Tolmatz, L. (2005). Method for constructing Stieltjes classes for M-indeterminate probability distributions. Appl. Math. Comput. 165, 669-685.

Williamson, M. AND Gaston, K. J. (2005). The lognormal distribution is not an appropriate null hypothesis for the species-abundance distribution. J. Animal Ecology 74, 409-422.

YAMAZAKI, H. AND LUECK, R. (1990). Why oceanic dissipation rates are not lognormal? J. Phys. Oceanography 20, 1907-1918. 